\title{
Genetic variation in the TAS2R38 taste receptor contributes to the oral microbiota in North and South European locations: a pilot study
}

\author{
Mari A. Sandell ${ }^{1,2^{*}}$ (D) and Maria Carmen Collado ${ }^{1,3^{*}}$ (i)
}

\begin{abstract}
Background: Microbial communities are influenced by environmental factors including host genetics. We investigated the relationship between host bitter taste receptor genotype hTAS2R38 and oral microbiota, together with the influence of geographical location.

Methods: hTAS2R38 polymorphisms and $16 \mathrm{~S}$ bacterial gene sequencing from oral samples were analyzed from a total of 45 healthy volunteers from different geographical locations.

Results: Genetic variation in the bitter taste receptor TAS2R38 reflected in the microbial composition of oral mucosa in Finnish and Spanish subjects. Multivariate analysis showed significant differences in the microbial composition between country and also dependent on taste genotype. Oral microbiota was shown to be more stable to the geographical location impact among AVI-homozygotes than PAV-homozygotes or heterozygotes (PAV/AVI).

Conclusion: Geographical location and genetic variation in the hTAS2R38 taste receptor impact oral mucosa microbial composition. These findings provide an advance in the knowledge regarding the interactions between taste receptor genes and oral microbiota. This study suggests the role of host-microbiota interactions on the food taste perception in food choices, nutrition, and eating behavior.
\end{abstract}

Keywords: TAS2R38 taste receptor, Oral microbiota, Genetic variation, Cross-cultural study, Geographical location, Food and nutrition

\section{Background}

Tasty food is more likely acceptable, but the importance of foods and their taste may differ in different consumers. Human sense of taste detects at least sweetness, saltiness, sourness, bitterness, and umami. The perception of bitterness is known to be the most complex taste sensation with 25 G-protein-coupled TAS2R receptors [1]. The genes coding the bitter TAS2R receptors are already well known by geneticists [2]. Differences in genotypes vary with respect to amino acid substitutions encoded at certain positions on some of the bitter receptor proteins. So far, the most studied human taste receptor in TAS2R bitter taste receptor family is TAS2R38.

\footnotetext{
* Correspondence: mari.sandell@utu.fi; mcolam@iata.csic.es

${ }^{1}$ Functional Foods Forum, University of Turku, FI-20014, Turku, Finland Full list of author information is available at the end of the article
}

The differences between humans have been linked with amino acids 49 (P/A), 262(A/V), and 296 (V/I) [3]. Among the eight possible haplotypes, the "sensitive" PAV and the "less sensitive" AVI represent the most frequent ones [4].

Functional genetic variation in TAS2R38 relevant to perception of bitter compounds or food has been reported in several studies [5-9]. In addition to perception, there appears to be a link between TAS2R38 genotype and vegetable consumption [10-12]. Higher intake of daily energy from sugars and candy among preschool-aged PAV/PAV boys has been suggested [13]. PAV/PAV children have also shown to prefer not only higher concentration of sugar solution, but also sweeter beverages and cereals than AVI/AVI children [6]. PAV homozygote adult males and females were found to

(c) The Author(s). 2018 Open Access This article is distributed under the terms of the Creative Commons Attribution 4.0 International License (http://creativecommons.org/licenses/by/4.0/), which permits unrestricted use, distribution, and 
consume more sweet foods such as soft drinks, sweets, chocolate, ice cream than AVI homozygotes [10]. Other TAS bitter receptor genes have been connected to perception of specific beverages [14]. At the moment, individual sensory worlds are only partly understood and need to be assessed in detail their implications for human well-being [15-17]. These findings suggest that genetic variation in taste receptors plays a role in both food preferences and consumption. Eating is also closely linked to daily life; people differ from each other in food preferences and food consumption.

It has been suggested that gut microbiome would be able to modulate human food preferences, nutritional intake, and also host eating behavior [18], but the evidence with oral microbiome is still uncovered. The human oral cavity is colonized by a wide range of microorganisms and composed by over 600 prevalent taxa at the species level $[19,20]$. It is known that human oral microbiome is affected by multiple factors, including diet, lifestyle, and antibiotics [21, 22]. Differences in oral microbiome have been reported depending on environments and climatic conditions [23], microbial diversity in dental calculus was lower in modern Europeans than Mesolithic or preindustrial Neolithic [24], meat consumption had an impact on the Neanderthal oral microbiome [25] and higher consumption of fermentable carbohydrates was linked to a reduced oral bacterial diversity [26]. However, little is known on the role of the human oral microbiome in food perception, processing and nutrition, or the effect of taste genotypes in the human oral microbiome.

In this situation, our main goal was to study the impact of TAS2R38 taste receptor haplotypes on human oral microbiota. We focused on three questions: (1) is there an association between TAS2R38 taste receptor and oral microbiota of humans, (2) is there a food cultural impact on differences of oral microbiota based on geographical origin of subjects, (3) is there a connection between genetic variation and functional activity of microbes. This translational study was carried out in northern and southern locations in Europe (Finland and Spain, respectively).

\section{Methods}

\section{Subjects and sampling}

A total of 45 healthy voluntary subjects (26 females and 19 males) from two European countries located in South (Spain, $n=23,12$ males and 11 females) and North Europe (Finland, $n=22,7$ males and 15 female) participated in the study. The participants' written informed consent was obtained. No antibiotics, medication, and probiotic consumption were reported. The study protocol was approved by the Ethical Committee of the Atencion
Primaria-Generalitat Valenciana (CEIC-APCV) and Ethical Committee of Southwest Finland Hospital District's.

The volunteers were given oral and written instructions for a punctual standardized collection of saliva samples in the morning ( $2 \mathrm{~h}$ after cleaning teeth and before eating any food and coffee). Cheek-swabs (Catch-All Sample Collection Swab ${ }^{\circ}$, Epicentre, USA) were used to collect buccal cells from the mouth of subjects and were kept frozen $-80^{\circ} \mathrm{C}$ until delivery to the laboratory.

\section{TAS2R38 genotyping}

hTAS2R38 polymorphisms of subjects were centralized and determined based on three SNPs (rs713598, rs1726866, and rs10246939) in Functional Foods Forum (University of Turku, Finland). DNA was extracted from swabs with QuickExtract DNA Extraction Solution ${ }^{\circ}$ following the instructions of the manufacturer (Epicenter, Madison, WI, USA). We used allele-specific probes and primers from Applied Biosystems (Foster City, CA, USA) to determine alleles (A49P, V262A, and 1296V). The genotyping were carried out with 7300 Real Time PCR system of Applied Biosystems [5-7].

\section{Pyrosequencing of tagged 16S rRNA gene amplicons}

A specific fragment of $500 \mathrm{bp}$ of the $16 \mathrm{~S}$ rRNA gene (500 bp from V1 to V3 region) was amplified (27F and 533R primers) using the high-fidelity polymerase (AB-Gene DNA, Thermo Scientific), as described previously [27]. Amplicons were checked and measured using the Agilent High Sensitivity DNA assay in Agilent 2100 Expert. Purified PCR products were sequenced from the forward-end using a 454 GS sequencer with Titanium chemistry (Roche) at FISABIO Center (Valencia, Spain).

\section{Bioinformatics and statistical analysis}

The raw data set was trimmed and filtered by quality (minimum average quality score of 20 ) and length sequence (minimum sequence length of $200 \mathrm{bp}$ ). All these steps were performed through the galaxy server (http:// getgalaxy.org/). Data processing was done by QIIME pipeline (version 1.9.0) [28], and sequences were further filtered to remove chimeric sequences [29]. Sequences were clustered into Operational Taxonomic Units (OTUs) at 97\% identity and then, taxonomy assignment was done by Greengenes $16 \mathrm{~S}$ rRNA gene database (Version 13.8). Sequences not assigned to bacterial kingdom and/or identified as cyanobacteria and chloroplasts were removed as they would represent ingested vegetal material and considered as contaminants. Alpha diversity indices (Chao1 and Shannon indexes) and beta diversity using UNIFRAC (phylogenetic) and Bray-Curtis distance (non-phylogenetic) among samples and PERMANOVA were used to test the differences. The significance level was set at $P<0.05$. 
Calypso software version 8.20 (http://cgenome.net/ calypso/) was used with total sum normalization (TSS) for the statistical analysis, and also, cumulative sum scaling normalization (CSS) for multivariate tests (redundancy analysis-RDA) and data mining. DESeq2 method was used to explore whether the groups were associated with altered bacterial relative abundances [30].

Predictive functional analysis was performed using PICRUSt [31] with Kyoto Encyclopedia of Genes and Genomes (KEGG) Pathway abundance classification. Biomarker identification (bacterial taxonomy and KEGG pathways) was analyzed by the Linear discriminant analysis Effect Size (LEfSe) test [32].

\section{Results}

\section{Subjects and sampling}

All the subjects were volunteer adults with age $36.28 \pm$ 5.72 (mean \pm SD) years old in the Spanish cohort and $41.00 \pm 13.10$ (mean \pm SD) year old in the Finnish cohort. Distribution in genetic variation of TAS2R38 taste receptor is shown in Table 1. One male volunteer in the Spanish cohort was classified in the AAV/AVI genotype, and the sample was removed from the whole analysis.

\section{Oral microbiota composition and diversity}

From 214,532 quality and length filtered sequences (average length $=385.6 \mathrm{bp}, \min =200 \mathrm{bp}$ and $\max =475 \mathrm{bp}$ ) were included in the study, and after chimera removal, a total of 212,077 sequences were analyzed (4819.9 \pm 1074.7 mean \pm SD sequences per sample). These sequences covered 5612 unique OTUs in whole population 44 subjects.

\section{Geographical location effect on oral microbiota profiles}

The geographical location had an impact on the oral microbiota as demonstrated by the multivariate method PERMANOVA with 999 permutation on the UniFrac distances (phylogenetic) $(P=0.034$ for Unweighted UNIFRAC distance) and Bray-Curtis distance (non-phylogenetic) $(P=0.006)$. Furthermore, multivariate redundant discriminant analysis (RDA) showed statistically significant differences in microbial composition between oral samples from different locations $(P=0.003)$ (Fig. 1a).

Higher abundance of Actinobacteria was observed in the Finnish samples compared to the Spanish ones $(5.96 \%$ vs

Table 1 Number of subjects in each genotype group from Spain and Finland

\begin{tabular}{llll}
\hline Genotype & Spain & Finland & Total \\
\hline AVI/AVI & $4(18.2 \%)$ & $7(31.8 \%)$ & $11(25.0 \%)$ \\
PAV/AVI & $14(63.6 \%)$ & $7(31.8 \%)$ & $21(47.7 \%)$ \\
PAV/PAV & $4(18.2 \%)$ & $8(32.4 \%)$ & $12(27.3 \%)$ \\
Total & 22 & 22 & $44(100 \%)$ \\
\hline
\end{tabular}

$2.36 \%$, respectively, $P=0.008)$. At family level, significant higher abundance of Paraprevotellaceae $(P=0.0035)$, Fusobacteriaceae $(P=0.041)$, Peptostreptococcaceae $(P=$ 0.011), unclassified Bacteroidales $(P=0.031)$ and Clostridiales $(P=0.016)$ and lower abundance of Micrococcaceae $(P=0.0008)$, Burkholderiaceae $(P=0.035)$, Gemellaceae $(P$ $=0.042)$ were detected in the Spanish samples compared to those abundance found in Finnish group (Fig. 1b).

Alpha diversity analysis showed not significant differences in diversity (Shannon) and richness (Chao1) between geographical locations (Fig. 1c). To test the geographical differences between countries, the DESeq2 test was used. The abundance of Rothia $(P=0.0009)$ and unclassified Gemellaceae $(P=0.0056$, ) was significantly higher in Finland than in Spain. Lactococcus, Fusobacterium, and Porphyromonas genus were significantly higher in Spain compared to that in Finland ( $P=0.0002, P=0.0021$, and $P=0.0011$, respectively).

Using Linear discriminant analysis (LDA) Effect Size (LEfSe) test, taxonomic differences between countries at genus level were found: Spanish oral microbiome was significantly enriched in Fusobacterium, while Rothia, Lautropia, and unclassified Gemellaceae were enriched in the Finnish samples (Fig. 1d).

\section{Taste genotype shapes the oral microbiota profiles in each location}

There are different associations between TAS2R38 taste receptor and oral microbiota profile depending on the geographical location. Significant differences in the oral microbial composition between taste genotypes and locations were found (RDA test, $P=0.046$ ) (Fig. 2a) and also, by multivariate method PERMANOVA with 999 permutation on Bray Curtis distance (non-phylogenetic, $P=0.031$ ).

Shifts on the relative abundances of specific bacterial groups at family level according to taste genotype were identified at each location (Fig. 1b). In the southern location, higher relative abundance of Staphylococcaceae family $(P=$ $0.023)$ and Staphylococcus genus $(P=0.026)$ was found in AVI/AVI group compared to those observed in PAV/PAV and PAV/AVI. No differences in alpha diversity were found. In the Finnish cohort, higher abundance of Corynebacteriaceae $(P=0.048)$ was related to PAV/PAV genotype. At genus level, higher relative abundance of unclassified Lachnospiraceae $(P=0.027)$ and Corynebacterium $(P=0.048)$ were associated to PAV/PAV genotype. At species level, higher relative abundance of unclassified Veillonella $(P=0.020)$ was observed in PAV/AVI while Veillonella dispar was abundant $(P$ $=0.070$ ) in AVI/AVI. No significant differences were found in alpha-diversity although PAV/AVI showed lower diversity (Shannon index) as observed in the other two genotypes.

When genotypes between countries were compared, we found a higher abundance of Bacteroidetes phylum $(P=0.002)$ in Spanish PAV/AVI-heterozygotes than in Finnish PAV/AVI. At family level, Spanish PAV/AVI 


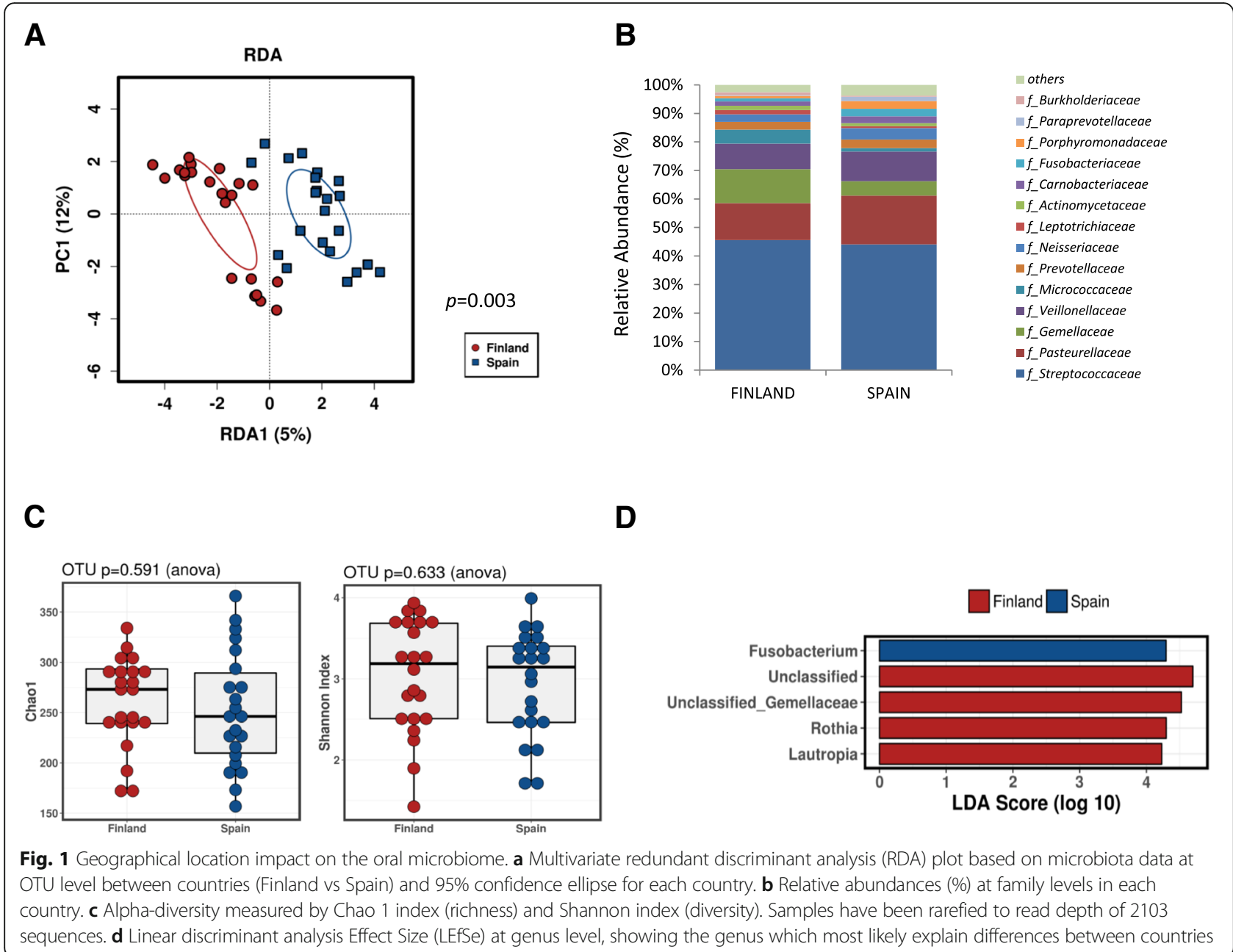

genotype had lower abundance of Gemellaceae $(P=$ $0.012)$ and Micrococcaceae $(P=0.048)$, and higher abundance of Lachnospiraceae $(P=0.0031)$, Prevotellaceae $(P$ $=0.038)$, Paraprevotellaceae $(P=0.040)$, and Corynebacteriaceae $(P=0.040)$ than in PAV/AVI Finnish samples. In PAV/PAV group, higher abundance of Actinobacteria and lower of Proteobacteria phylum where found in Finland than those observed for same genotype in the Spanish group $(P=0.004$ and $P=0.063$, respectively). At family level, we found significant higher abundance of Micrococcaceae $(P=0.008)$ and lower of Pasteurellaceae $(P$ $=0.028)$ and Staphylococcaceae $(P=0.037)$ in Finnish PAV/ PAV than in Spanish PAV/PAV. Spanish AVI-homozygotes harbored higher abundance of specific families as Peptostreptococcaceae $(P=0.016)$ and lower of Gemellaceae $(p=$ $0.062)$ and Fusobacteriaceae $(P=0.073)$ compared to those observed in Finnish AVI-homozygotes (Fig. 2b).

In detail, RDA analysis showed differences in the microbial composition PAV/PAV genotype and country $(P=$ 0.050) and also, same observation was found for PAV/AVI genotype $(P=0.019)$ while no effect of country was found in AVI/AVI genotype (also less sensitive in some taste) (Fig. 2c). These results suggest that AVI/AVI genotype (insensitive) microbiome is more stable to the environmental factors than the sensitive genotypes (PAV/AVI and PAV/ PAV). No differences in alpha-diversity were found although Finnish PAV/PAV genotype showed higher diversity (Shannon index) than Spanish PAV/PAV $(P=0.064)$.

\section{Predictive functional profiling of oral microbiota}

There is a connection between genetic variation in TAS2R38 and microbial functional activity. The PICRUSt prediction revealed significant differences in the main functional classes (Kyoto Encyclopedia of Genes and Genomes-KEGG categories at level 2), deriving from functional acquisitions associated with different locations (multivariate RDA test, $P=0.038$ ) and taste genotype per location (RDA test $P=0.048$, Fig. 3a).

The predominant activities in both locations are mostly represented by Bacterial chemotaxis, Zeatin 

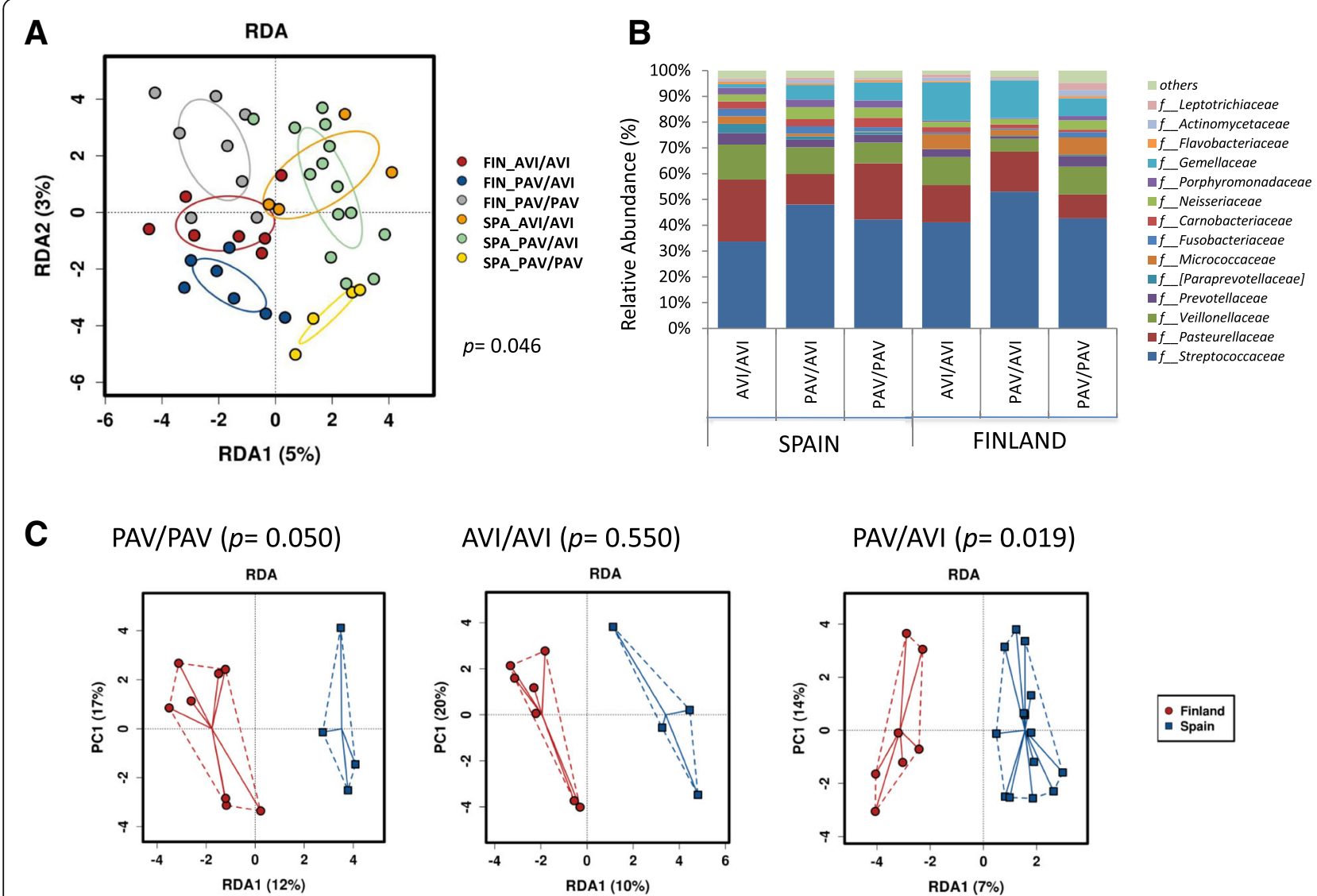

- Finland
- Spain

Fig. 2 Association between taste genotype, country, and oral microbiome. a RDA plots based microbiota analysis at OTU level depending on country and taste genotype (1:AVI/AVI, 2:PAV/AVI, and 3:PAV/PAV). b Relative abundances (\%) in each specific TAS2R38 taste receptor genotype and country. c RDA plots at OTU level on each genotype for country effect. Ellipses represent 95\% confidence intervals from centroids of the interaction between genotypes

biosynthesis, Cell motility, and secretion; and Phosphotransferase system PTS. The DAPC test identifies specific clusters of functional activity (KEGG level 2) of the oral microbiome in oral groups according to taste genotype and countryside (Fig. $3 \mathrm{~b}$ ) suggesting a distinct KEGG profile between groups although Spain AVI/AVI and PAV/PAV showed similar activities (both clustered together). Phosphotransferase system PTS, secretion system, and oxidative phosphorylation were the strongest indicator of the presence of distinct KEGG clusters.

LEfSe analysis performed on PICRUSt output showed several KEGG categories differentially present in the groups (Fig. 3c). In particular, in Finland group, we observed enrichment in phosphotransferase system PTS in PAV/AVI while fatty acid metabolisms and also Phreniltranferesae and aminoacid degradation were enriched in $\mathrm{PAV} / \mathrm{PAV}$. However, we found that bacterial secretion system was enriched in PAV/AVI Spanish samples while glycosyltransferases, phenylalanine, tyrosine, and tryptophan biosynthesis and translation of proteins were highly present in AVI/AVI genotype.

\section{Discussion}

Oral microbiota is a complex and dynamic bacterial community in which specific species and strains are competing for nutrients and mucosal adhesion sites in order to survive and multiply. The food eaten by the human host is considered the important factors shaping human microbiota. The role of genetic variation in taste receptors and moreover in our eating habits is currently under research around the world. Food-related studies have been focused on TAS2R38 so far, but this kind of multidisciplinary field of science combining genetics and oral microbiota is a hitherto unexplored area. To our knowledge, our pilot study represents the first report demonstrating a key interaction between taste genes and oral microbiome according to geographical location that would be linked to food choice covering the aspects of culture, availability, and preferences.

Our study included samples from Southern Europe (Spain) and Northern Europe (Finland) including also natural differences in food cultures and daily life practices related to eating habits. We found a strong influence of country on oral cavity microbiota. Although we did not collect dietary 


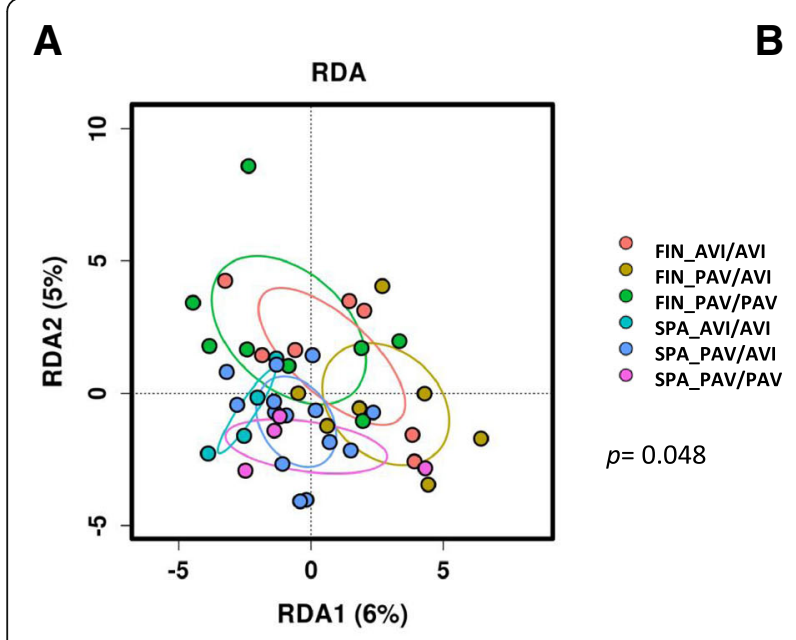

B
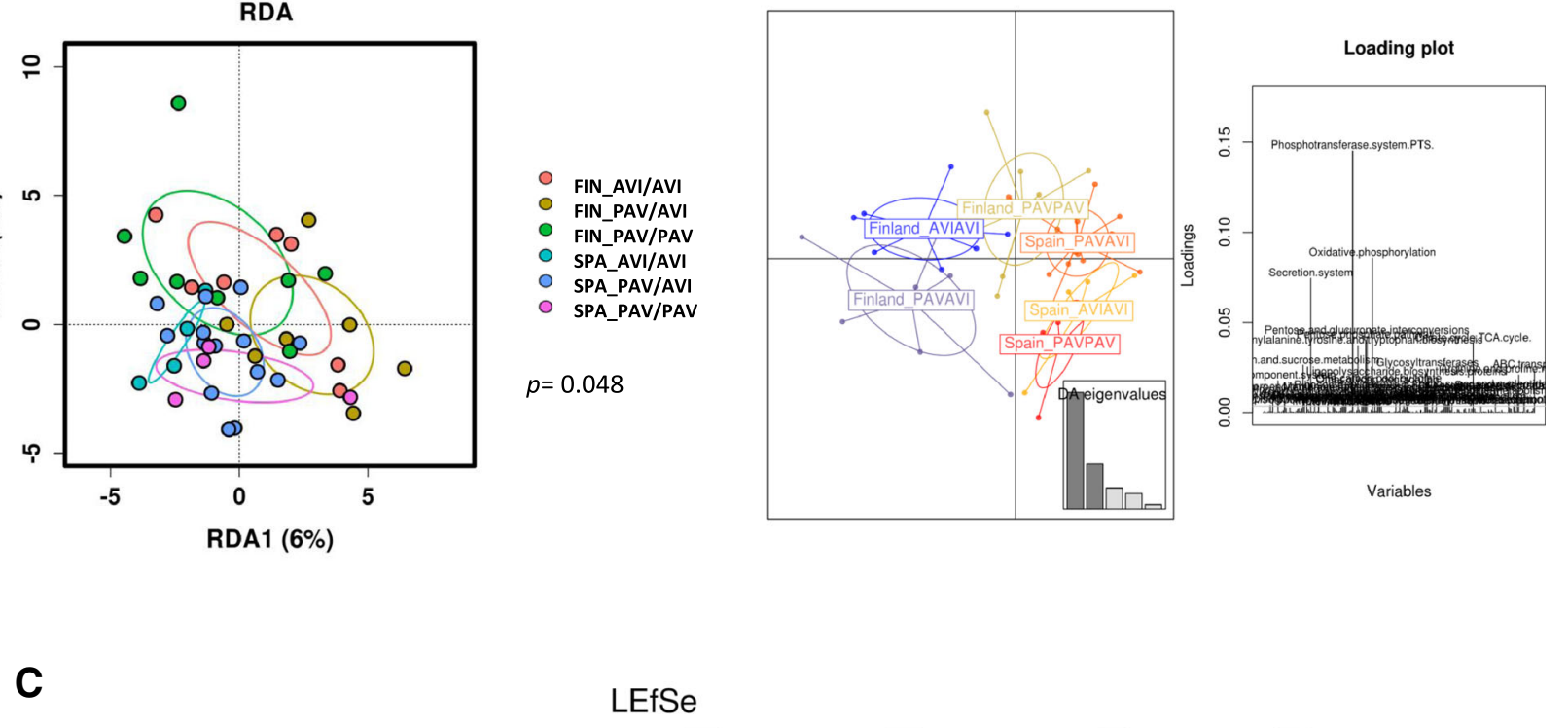

Finland_PAVAVI $\square$ Finland_PAVPAV $\square$ Spain_AVIAVI $\square$ Spain_PAVAVI

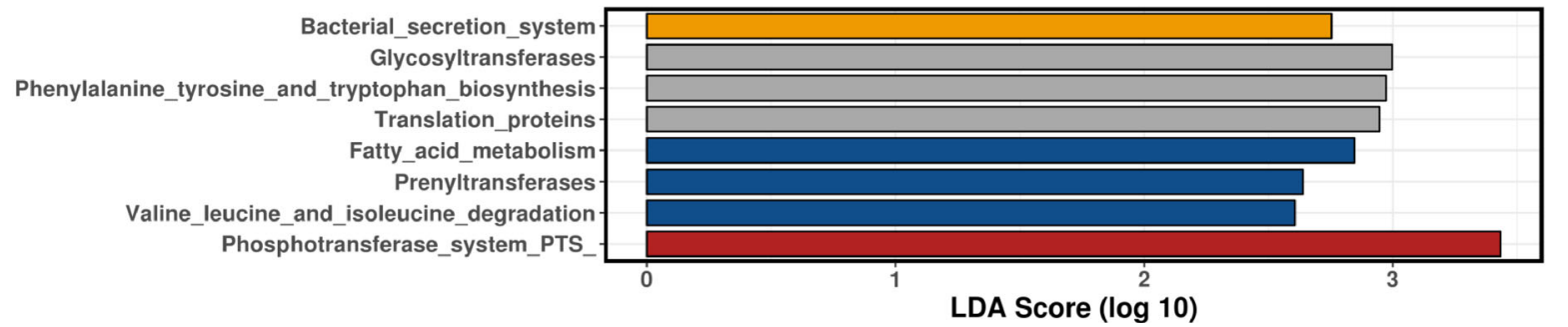

Fig. 3 Abundant pathways in the oral microbiome according to taste genotype and country. a RDA plots based on KEGG pathways (level 2), ellipses represent 95\% confidence intervals from centroids of the interaction between pathways. b Discriminant analysis of principal components (DAPC) plot at KEGG pathways (level 2). The individual peaks in the loading plot show the variable influence on the cluster separation (0.05 threshold level). c LEfSE test revealed distinct KEGG pathways in taste and country combination

information, there would be clear differences in diet and lifestyle between Spanish and Finnish subjects. Food culture may explain some differences found in oral microbiota composition.

It is well-established that genetic background and environment have a pivotal role on the microbiome. Geography has been shown to have a strong influence on the composition of microbial populations including gut and oral microbiota [33]. Human microbiome research has reported the relevance the geographic location (inter-country variation) in the context of the individual variation on the gut microbiome [34]. Gut microbes differ between healthy Amerindian, Malawian, and USA metropolitan inhabitants [35] but also, differences in saliva microbiome of human groups living in Africa, Alaska, and Germany (different environments and weather climatic conditions) have been reported [23]. Indeed, dysbiosis of the oral microbiome (and a leaky oral mucosa) might be key underlying imbalances in pathogenesis of specific diseases including autoimmune, inflammatory, and also cardiovascular diseases [19].

Our data reported that Rothia, Lautropia, and Gemellaceae were enriched in Finland samples while Fusobacterium and Porphyromonas genus were significantly higher in Spain compared to Finland. Both Rothiaspecies, Rothia mucilaginosa $(P=0.00001)$ and Rothia dentocariosa $(P=0.0024)$ have been more frequently in Finland than that in Spain. The higher abundance of Rothia, Lautropia, and Gemella have been more frequently reported as a core composition in the oral bacterial community in health [36, 37] while higher Fusobacterium and Porphyromonas have been related to risk of diseases [38]. It has been reported that an increase of Porphyromonas gingivalis would affect the oral microbiota equilibrium, favoring that other bacteria as Fusobacterium nucleatum would become opportunistic pathogen and the combined effect would increase the risk of periodontal disease [39]. 
Differences in microbiome between different geographical locations may be related to diet (e.g., quality and quantity) and lifestyle (e.g., time for eating, daily eating rhythm, eating site choice) although these connections have not been widely studied. Despite the country-based differences, a core of common bacteria is shared between countries formed mainly, in order of abundance, by Streptococcus, Veillonella, Gemellaceae, Rothia, Prevotella, Neisseria, Granulicatella, Actinomyces, Fusobacterium, Lautropia, Leptotrichia, Porphyromonas, Neisseriaceae, Actinobacillus, Campylobacter, Aggregatibacter, Weeksellaceae, Kingella, Gemella, TM7, Abiotrophia, Atopobium, Selenomonas, Staphylococcus, Corynebacterium, and Tannerella as previously reported in other studies [40].

It is known that taste perception has an impact on food preferences and food habits [41]. It has been also suggested that gut microbiome would be able to modulate human food preferences and also host eating behavior [18], but little is known on oral microbiota. This study focused on TAS2R38 taste receptor, which has a link to both selected bitter tasting compounds and food such as specific vegetables and sweet foods. The connection between food, mouth, oral cavity, and composition of microbiota is rational, although the supporting data available are very limited. Moreover, the individual differences in genetic variation of the human taste receptor such as TAS2R38 and in oral microbiome profile create a novel context and basis to explore the connection between host-microbe interactions and health. We found oral microbiota differences according to TAS2R38 taste receptor genotype in both geographical locations. Staphylococcaceae and Tissierellaceae were linked to Spanish AVI/AVI while Corynebacteriaceae were linked to Finnish PAV/PAV genotype. In both locations, no differences in microbial diversity and richness between genotypes were found.

Also our data is demonstrating the effect of diet on oral microbiota and supporting the results published before. It has been reported that omnivore, ovo-lacto-vegetarian, and vegan diet have an impact on the salivary metabolome but not in oral microbiota [42]. However, other study reported that Italian-style gluten-free diet was able to modulate both oral microbiota and metabolome of African celiac children [43]. Although dietary recalls or food consumption data were not collected in our study, as an approximation, we have compared the food intakes between the countries (among women) based on the national diet surveys carried out in Spain (Generalitat Valenciana survey 2010-2011, $n=1607,16-64$ years) [44] and Finland (Findiet 2012 survey, $n=710,25-64$ years) [45]. In Spain, the daily intake of vegetables, fruit, legumes, and potatoes was on average $444 \mathrm{~g}$, while in Finland, the daily intake of vegetables, root vegetables, legumes, fruits, berries, or juices was on average $400 \mathrm{~g}$ correspondingly. The fiber intake is higher in Finland (21 g/day) than in Spain (16 g/ day), as well as the fat intake (Finland $71 \mathrm{~g} /$ day, Spain $64 \mathrm{~g} /$ day). Daily consumption of fish dishes is $95 \mathrm{~g} /$ day in Finland, and $41.6 \mathrm{~g} /$ day (Spain).

Differences in diet and nutrition would explain partially the oral microbiome differences that we found between countries. Moreover, our results also showed a significant and distinct oral microbiome composition depending on taste genotype and country, suggesting a combined effect of environment-genetics-eating habits/preferences. Multivariate analysis showed significant differences in the oral microbiome of PAV/PAV and PAV/AVI taste genotype between countries while no country-differences were associated with AVI/AVI genotype. These pilot results suggest that AVI/AVI genotype microbiota may be more stable to environmental effects. Based on food or taste perception studies AVI/AVI homozygotes may be less sensitive in selecting bitter taste food items than PAV/PAV and also more flexible in food choices. It could be possible that environmental location and food culture will have milder effect on AVI/AVI than on PAV/PAV, because AVI/AVI may accept high variability on diet as it has been suggested that insensitive (AVI/AVI) may be more flexible than PAV/ PAV in their food selection. There are studies showing the connection between TAS2R38 and higher content of vegetables and sweet food, but there are also studies showing no effect on intake of vegetables, fat, or micronutrients $[16,46]$. Those conflicting findings related to TAS2R38 and food consumption may be explained by different variety at the local food culture or the methods used to evaluate dietary intake. This may also explain the reason why we did not find significant country effect on AVI/AVI hosts carrying oral cavity microbiome either in Spain or in Finland, and higher differences in taste receptor genotypes. Moreover, this kind of multidisciplinary research combining genetics and eating behavior is still pretty new.

We found higher abundance of Peptostreptococcaceae and lower of Gemellaceae in AVI/AVI genotype between Spain than those observed in Finland. Peptostreptococcacee family has been described to have an inverse relationship with an inflammation marker C-reactive protein (CRP) [47]. Furthermore, it has been reported that AVI/AVI genotype is linked to obesity and inflammatory-related problems [48]. TAS2R38 polymorphism has been also associated with susceptibility to respiratory tract infections [49]. We also found significant higher abundance of Bacteroidetes phylum in PAV/AVI genotype. It has been reported that a higher Bacteroidetes and lower Proteobacteria abundances in the salivary microbiota have been linked to IBD patients who also showed a higher pro-inflammatory profile (higher pro-inflammatory cytokines and higher-immunoglobulin A levels) compared to control group [50]. Significant differences in oral microbiome composition and diversity between Finland and Spain may be partially explained by food availability and food culture and tradition. PAV/PAV 
genotype was linked to significantly higher Actinobacteria and lower Proteobacteria, as well as lower abundance of Pasteurellaceae and Staphylococcaceae in Finland compared to Spain.

These new findings call for future studies to reveal the consequences of taste receptor polymorphisms and microbiota on human health in the long run. Research focusing on microbiota or sensory receptors alone is still needed to understand the molecular mechanisms and bioscientific models, but methods should be interconnected to learn more about human behavior.

This pilot study has some limitations that include sample size in each country and taste genotypes group size which would have affect the statistical power of the study. Dietary records were not collected, and no association with food choice and preferences could be studied. Furthermore, the pyrosequencing method has been replaced by new methodologies with higher coverage and higher number of sequences per sample. However, despite those limitations and the fact that further research is needed, our results indicate that taste genotype affects oral microbial diversity and microbial community composition. Our study provides new insights on the link between oral microbiota-taste genotype, and food may promote further research in the field of dietary strategies, microbiota, and health.

\section{Conclusions}

Taste receptor genotype has an impact on human oral microbiome via geographical location. Although our study provides information on the role of the taste genes on the microbiome and food culture, further research is needed to explore the complex and dynamic interrelation between diet-genotype-microbiome and health. Deep understanding of the impact of environmental factors as geographical location, diet, and lifestyle on the human microbiota would provide future personalized dietary strategies to modulate microbiota aimed to reduce the risk of microbial dysbiosis linked to high risk of non-communicable diseases as obesity, diabetes, and allergy.

\section{Abbreviations \\ AVI: Alanine, valine, isoleucine (a haplotype presenting polyphormisms in the TAS2R38 taste receptor gene A49P, V262A, 1296V); AVI/AVI: Homozygotic diplotype composed by two AVI-haplotypes; PAV: Proline, alanine, valine (a haplotype presenting polyphormisms in the TAS2R38 taste receptor gene A49P, V262A, 1296V); PAV/AVI: Heterozygotic genotype composed by haplotypes PAV and AVI; PAV/PAV: Homozygotic diplotype composed by two PAV-haplotypes;} TAS2R38: One of the bitter taste receptors

\section{Acknowledgements}

We thank Seppo Salminen and Paul A.S. Breslin for their scientific advices. We also thank Hilkka Terho, Saila Mattila, and Izaskun Garcia-Mantrana for their technical help. We are grateful for all the study participants providing oral cavity sample for this study.

\section{Funding}

This study was funded by Academy of Finland (MS252005 and 309408), University of Turku (Faculty of Medicine). MCC would like to acknowledge the i-LINK CSIC grant (I-LINK0679).

\section{Availability of data and materials}

The datasets used for analysis during the current study are not publicly available but available from the corresponding author on reasonable request.

\section{Authors' contributions}

MS and MCC formulated the research questions and designed the research, performed statistical analysis, contributed to the interpretation of results and revision of the manuscript, and wrote the paper. All authors read and approved the final version.

\section{Ethics approval and consent to participate}

The study protocol was approved by the Ethical Committee of the Atencion Primaria-Generalitat Valenciana (CEIC-APCV) and Ethical Committee of Southwest Finland Hospital District's. All participants have given their written informed consent.

Consent for publication

Not applicable

\section{Competing interests}

The authors declare that they have no competing interests.

\section{Publisher's Note}

Springer Nature remains neutral with regard to jurisdictional claims in published maps and institutional affiliations.

\section{Author details}

${ }^{1}$ Functional Foods Forum, University of Turku, Fl-20014, Turku, Finland. ${ }^{2}$ Monell Chemical Senses Center, Philadelphia, USA. ${ }^{3}$ Department of Biotechnology, Institute of Agrochemistry and Food Technology, Spanish National Research Council (IATA-CSIC), Av. Agustin Escardino 7, 46980 Valencia, Spain.

Received: 22 May 2018 Accepted: 24 October 2018

Published online: 04 December 2018

\section{References}

1. Kim UK, Breslin PAS, Reed D, Drayna D. Genetics of human taste perception. J Dent Res. 2004;83(6):448-53.

2. Meyerhof W, Batram C, Kuhn C, Brockhoff A, Chudoba E, Bufe B, Appendino $\mathrm{G}$, Behrens $\mathrm{M}$. The molecular receptive ranges of human TAS2R bitter taste receptors. Chem Senses. 2010:35(2):157-70.

3. Kim UK, Drayna D. Genetics of individual differences in bitter taste perception: lessons from the PTC gene. Clin Genet. 2005:67(4):275-80.

4. Risso DS, Mezzavilla M, Pagani L, Robino A, Morini G, Tofanelli S, Carrai M, Campa D, Barale R, Caradonna F, Gasparini P, Luiselli D, Wooding S, Drayna D. Global diversity in the TAS2R38 bitter taste receptor: revisiting a classic evolutionary PROPosal. SciRep. 2016;6:25506.

5. Bufe B, Breslin PAS, Kuhn C, Reed DR, Tharp CD, Slack JP, Kim UK, Drayna D, Meyerhof $\mathrm{W}$. The molecular basis of individual differences in phenylthiocarbamide and propylthiouracil bitterness perception. Curr Biol. 2005;15(4):322-7.

6. Mennella JA, Pepino MY, Reed DR. Genetic and environmental determinants of bitter perception and sweet preferences. Pediatrics. 2005;115:e216-22.

7. Sandell M, Breslin PAS. Variability in a taste-receptor gene determines whether we taste toxins in food. Curr Biol. 2006;16(18):R792-4.

8. Laaksonen $\mathrm{O}$, Ahola J, Sandell M. Explaining and predicting individually experienced liking of berry fractions by the hTAS2R38 taste receptor genotype. Appetite. 2013;61:85-96.

9. Feeney $\mathrm{E}$. The impact of bitter perception and genotypic variation of TAS2R38 on food choice. Nutr Bull. 2011;36(1):20-33.

10. Sandell M, Hoppu U, Mikkilä V, Mononen N, Kähönen M, Männistö S, Rönnemaa T, Viikari J, Lehtimäki T, Raitakari OT. Genetic variation in the hTAS2R38 taste receptor and food consumption among Finnish adults. Genes Nutr. 2014;9:433. 
11. Duffy VB, Hayes JE, Davidson AC, Kidd JR, Kidd KK, Bartoshuk LM. Vegetable intake in college-aged adults is explained by oral sensory phenotypes and TAS2R38 genotype. Chemosens Percept. 2010;3(3-4):137-48.

12. Gorovic N, Afzal S, Tjønneland A, Overvad K, Vogel U, Albrechtsen C, Poulsen HE. Genetic variation in the hTAS2R38 taste receptor and brassica vegetable intake. Scand J Clin Lab Invest. 2011;71(4):274-9.

13. Hoppu U, Laitinen K, Jaakkola J, Sandell M. The hTAS2R38 genotype is associated with sugar and candy consumption in preschool boys. J Hum Nutr Diet. 2015;1:45-51.

14. Hayes JE, Wallace MR, Knopik VS, Herbstman DM, Bartoshuk LM, Duffy VB. Allelic variation in TAS2R bitter receptor genes associates with variation in sensations from and ingestive behaviors toward common bitter beverages in adults. Chem Senses. 2011:36(3):311-9.

15. Hayes JE, Feeney EL, Allen AL. Do polymorphisms in chemosensory genes matter for human ingestive behavior? Food Qual Prefer. 2013;30(2):202-16

16. Hoppu U, Knaapila A, Laaksonen O, Sandell M. Genetic basis of flavor sensitivity and food preferences. In flavor: from food to behaviors, wellbeing and health. Woodhead publishing series in food science, technology. Nutrition. 2016;299:203-27.

17. Tepper BJ, Koelliker Y, Zhao L, Ullrich NV, Lanzara C, d'Adamo P, Ferrara A, Ulivi S, Esposito L, Gasparini P. Variation in the bitter-taste receptor gene TAS2R38, and adiposity in a genetically isolated population in southern Italy. Obesity. 2008;16(10):2289-95.

18. Alcock J, Maley CC, Aktipis CA. Is eating behavior manipulated by the gastrointestinal microbiota? Evolutionary pressures and potential mechanisms. BioEssays. 2014;36(10):940-9.

19. Seymour GJ, Ford PJ, Cullinan MP, Leishman S, Yamazaki K. Relationship between periodontal infections and systemic disease. Clin Microbiol Infect. 2007;13(Suppl 4):3-10.

20. Sakamoto M, Umeda M, Benno Y. Molecular analysis of human oral microbiota. J Periodontal Res. 2005;40:277-85.

21. Dagli N, Dagli R, Darwish S, Baroudi K. Oral microbial shift: factors affecting the microbiome and prevention of oral disease. J Contemp Dent Pract. 2016;17(1):90-6.

22. Kilian M, Chapple IL, Hannig M, Marsh PD, Meuric V, Pedersen AM, Tonetti MS, Wade WG, Zaura E. The oral microbiome-an update for oral healthcare professionals. Br Dent J. 2016;221(10):657-66.

23. Li J, Quinque D, Horz HP, Li M, Rzhetskaya M, Raff JA, Hayes MG, Stoneking M. Comparative analysis of the human saliva microbiome from different climate zones: Alaska, Germany, and Africa. BMC Microbiol. 2014;14:316.

24. Adler CJ, Dobney K, Weyrich L, Kaidonis J, Walker A, Haak W, Bradshaw J, Townsend G, Sołtysiak A, Alt KW, Parkhill J, Cooper A. Sequencing ancient calcified dental plaque shows changes in oral microbiota with dietary shifts of the neolithic and industrial revolutions. Nat Genet. 2013;45:450-5.

25. Weyrich LS, Duchene S, Soubrier J, Arriola L, Llamas B, Breen J, et al. Neanderthal behaviour, diet, and disease inferred from ancient DNA in dental calculus. Nature. 2017:544(7650):357-61.

26. Ribeiro AA, Azcarate-Peril MA, Cadenas MB, Butz N, Paster BJ, Chen T, Bair E, Arnold RR. The oral bacterial microbiome of occlusal surfaces in children and its association with diet and caries. PLoS One. 2017;12(7):e0180621.

27. Simón-Soro A, Tomás I, Cabrera-Rubio R, Catalan MD, Nyvad B, Mira A Microbial geography of the oral cavity. J Dent Res. 2013;92(7):616-21.

28. Caporaso JG, Kuczynski J, Stombaugh J, Bittinger K, Bushman FD, Costello EK, et al. QIIME allows analysis of high-throughput community sequencing data. Nat Methods. 2010;7(5):335-6.

29. Edgar RC, Haas BJ, Clemente JC, Quince C, Knight R. UCHIME improves sensitivity and speed of chimera detection. Bioinformatics. 2011;27(16): 2194-200.

30. Love Ml, Huber W, Anders S. Moderated estimation of fold change and dispersion for RNA-seq data with DESeq2. Genome Biol. 2014;15(12):550

31. Langille MG, Zaneveld J, Caporaso JG, McDonald D, Knights D, Reyes JA, Clemente JC, Burkepile DE, Vega Thurber RL, Knight R, Beiko RG, Huttenhower C. Predictive functional profiling of microbial communities using $16 \mathrm{~S}$ rRNA marker gene sequences. Nat Biotechnol. 2013;31(9):814-21.

32. Segata N, Izard J, Waldron L, Gevers D, Miropolsky L, Garrett WS, Huttenhower C. Metagenomic biomarker discovery and explanation. Genome Biol. 2011;12(6):R60.

33. Gupta VK, Paul S, Dutta C. Geography, ethnicity or subsistence-specific variations in human microbiome composition and diversity. Front Microbiol. 2017:8:1162.

34. Lloyd-Price J, Abu-Ali G, Huttenhower C. The healthy human microbiome. Genome Med. 2016;8:51.
35. Yatsunenko T, Rey FE, Manary MJ, Trehan I, Dominguez-Bello MG, Contreras $M$, et al. Human gut microbiome viewed across age and geography. Nature. 2012;486:222-7.

36. Abusleme L, Dupuy AK, Dutzan N, Silva N, Burleson JA, Strausbaugh LD, Gamonal J, Diaz PI. The subgingival microbiome in health and periodontitis and its relationship with community biomass and inflammation. ISME J. 2013;7:1016-25

37. Kistler JO, Booth V, Bradshaw DJ, Wade WG. Bacterial community development in experimental gingivitis. PLoS One. 2013;8(8):e71227.

38. Whitmore SE, Lamont RJ. Oral Bacteria and cancer. PLOS Pathog. 2014;10(3): e1003933.

39. Hajishengallis G, Lamont RJ. Beyond the red complex and into more complexity: the polymicrobial synergy and dysbiosis (PSD) model of periodontal disease etiology. Mol Oral Microbiol. 2012;27:409-419.39.

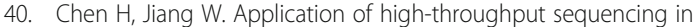
understanding human oral microbiome related with health and disease. Front Microbiol. 2014;5:508.

41. García-Bailo B, Toguri C, Eny KMA, El-Sohemy A. Genetic variation in taste and its influence on food selection. OMICS. 2009;13(1):69-80.

42. De Filippis F, Vannini L, La Storia A, Laghi L, Piombino P, Stellato G, et al. The same microbiota and a potentially discriminant metabolome in the saliva of omnivore, ovo-lacto-vegetarian and vegan individuals. PLoS One. 2014;9(11):e112373.

43. Ercolini D, Francavilla R, Vannini L, De Filippis F, Capriati T, Di Cagno R, lacono G, De Angelis M, Gobbetti M. From an imbalance to a new imbalance: Italian-style gluten-free diet alters the salivary microbiota and metabolome of African celiac children. Sci Rep. 2015;5:18571.

44. Quiles J, Jiménez R, Marín S, Rizk J, Zubeldía L. Encuesta de nutrición de la Comunitat Valenciana 2010-2011: Informe reducido. Valencia: Generalitat. Conselleria de Sanitat; 2013.

45. Helldán A, Raulio A, Kosola M, Tapanainen H, Ovaskainen M-L, Virtanen S. The National FINDIET 2012 Survey. Helsinki: THL. Report. 16/2013, 187 s; 2013.

46. Feeney E, O'Brien S, Scannell A, Markey A, Gibney R. Genetic variation in taste perception: does it have a role in healthy eating? Proc Nutr Soc. 2011; 70(1):135-43.

47. Hansen TH, Gobel RJ, Hansen T, Pedersen O. The gut microbiome in cardiometabolic health. Genome Med. 2015:7:33.

48. Ortega FJ, Agüera Z, Sabater M, Moreno-Navarrete JM, Alonso-Ledesma I, Xifra G, et al. Genetic variations of the bitter taste receptor TAS2R38 are associated with obesity and impact on single immune traits. Mol Nutr Food Res. 2016;60(7):1673-83.

49. Lee RJ, Xiong G, Kofonow JM, Chen B, Lysenko A, Jiang P, et al. T2R38 taste receptor polymorphisms underlie susceptibility to upper respiratory infection. J Clin Invest. 2012:122:4145-59.

50. Said HS, Suda W, Nakagome S, Chinen H, Oshima K, Kim S, et al. Dysbiosis of salivary microbiota in inflammatory bowel disease and its association with oral immunological biomarkers. DNA Res. 2014;21:15-25.

Ready to submit your research? Choose BMC and benefit from:

- fast, convenient online submission

- thorough peer review by experienced researchers in your field

- rapid publication on acceptance

- support for research data, including large and complex data types

- gold Open Access which fosters wider collaboration and increased citations

- maximum visibility for your research: over $100 \mathrm{M}$ website views per year

At $\mathrm{BMC}$, research is always in progress.

Learn more biomedcentral.com/submissions 